\section{Pneumatic retinopexy in the treatment of primary rhegmatogenous retinal detachment}

\begin{abstract}
Purpose To review the management by pneumatic retinopexy of $\mathbf{3 1}$ primary rhegmatogenous retinal detachments performed between August 1994 and December 1997. Methods Ocular indications included superior retinal breaks, no evidence of proliferative vitreoretinopathy (PVR) and ability to posture. Patients with inferior breaks and/or areas of vitreoretinal degeneration were excluded. Surgery was performed under local anaesthetic using sulphur hexafluoride $\left(\mathrm{SF}_{6}\right)$ or perfluoropropane $\left(\mathrm{C}_{3} \mathrm{~F}_{8}\right)$ gas injection. Transconjunctival cryotherapy or laser retinopexy was used to create permanent retinal adhesion. The mean length of patient follow-up was 11 months (range 5-24 months). Results Thirty-one patients (20 men, 11 women) with a mean age of 63.4 years (range 29-81 years) underwent pneumatic retinopexy which resulted in initial retinal reattachment in 22 patients. Two detachments recurred in the first

retinal detachments with retinal breaks in the superior periphery. A gas bubble is injected into the vitreous cavity and the patient is positioned so that the bubble tamponades the retinal break. Photocoagulation and/or cryotherapy are used to form a permanent adhesion around the break. Over the past 15 years a variable success rate of reattachment $\left(53-84 \%^{2-9}\right)$ after pneumatic retinopexy has been reported and several authors have extended its indications to large and giant tears, multiple breaks in multiple quadrants and detachments associated with a moderate degree of proliferative vitreoretinopathy (PVR) ${ }^{10-12}$ Controversy remains as to the application of this procedure in pseudophakic eyes with an intact or breached posterior capsule. ${ }^{4,13-15}$

This series consists of 31 cases of pneumatic retinopexy performed for primary rhegmatogenous retinal detachment at two hospitals between August 1994 and December 1997.
\end{abstract} month and a third at 4 months post-operatively, giving an anatomical reattachment rate with one procedure in 19 out of 31 eyes $(61 \%)$. Of the 12 failures, 7 were reattached with one additional operation and one case reattached after multiple procedures, giving an overall reattachment rate of $87 \%$. Post-operatively, new or missed breaks were present in 7 patients (22\%) and PVR developed in 4 patients (13\%). There was no difference in age, gender or extent of detachment between the failed and reattached groups and pseudophakia did not appear to be a poor prognostic factor. Conclusion Pneumatic retinopexy can be a useful alternative to conventional rhegmatogenous retinal detachment surgery in carefully selected cases. A larger study addressing the influence of non-ocular factors is warranted.

Key words Pneumatic retinopexy, Rhegmatogenous retinal detachment

Pneumatic retinopexy is a term introduced by Hilton and Grizzard in $1986^{1}$ to describe a treatment for selected primary rhegmatogenous
Patients and methods

A proportion of patients presenting with primary rhegmatogenous retinal detachment and deemed suitable for pneumatic retinopexy were offered that treatment. Ocular indications were breaks in the upper retinal quadrants, no evidence of PVR, no previous vitreoretinal surgery and ability to posture. Additional selection criteria included patients' general health and their unfitness for and/or unavailability of immediate anaesthetic cover and operating theatre time. Patients with inferior retinal breaks and/or areas of vitreoretinal degeneration were excluded. The patients understood the procedure and the need for post-operative positioning.

Surgery was performed under local anaesthesia in sterile conditions. The surface of the eye was treated with povidone-iodine solution and firm pressure was applied to the globe in order to reduce the intraocular pressure. Under indirect ophthalmoscopic control, sulphur hexafluoride $\left(\mathrm{SF}_{6}\right)$ or
A.C. Assi

D.G. Charteris

Z.J. Gregor

Moorfields Eye Hospital

City Road

London EC1V 2PD, UK

A.C. Assi

R.V. Pearson

Southend Hospital

Westcliff-on-Sea

Essex SSO ORY, UK

Mr A. Assi, BSC,

FRCOphth

Moorfields Eye Hospital

City Road

London EC1V 2PD, UK

Tel: +44 (0)171 5662285

Fax: $+44(0) 1712534696$

Received: 12 April 1999 Accepted in revised form: 24 June 1999 
Table 1. Operative procedures (31 eyes)

\begin{tabular}{lc}
\hline Procedure & No. of eyes \\
\hline Sulphur hexafluoride gas injection & \\
$\quad$ (mean volume $0.44 \mathrm{ml}$ ) & 8 \\
Perfluoropropane gas injection & 23 \\
$\quad$ (mean volume $0.32 \mathrm{ml}$ ) & 18 \\
Cryotherapy and gas injection on the same day & 5 \\
Cryotherapy 1-4 days after gas injection & 8 \\
Laser 1-4 days after gas injection & \\
\hline
\end{tabular}

perfluoropropane $\left(\mathrm{C}_{3} \mathrm{~F}_{8}\right)$ gas was briskly injected through the pars plana $4 \mathrm{~mm}$ posterior to the limbus and away from the break, with the patient's head turned to make the injection site uppermost. The gas was injected with only 2-3 $\mathrm{mm}$ of the 30 -gauge needle tip inside the vitreous cavity to produce a single gas bubble. Transconjunctival cryotherapy or laser retinopexy was used to create a retinal adhesion around the break. In cases of bullous detachment, retinopexy was applied post-operatively once the retina reattached.

The central retinal artery was monitored for $10 \mathrm{~min}$ and an anterior chamber paracentesis performed to restore retinal circulation if necessary. The surgical procedures are summarised in Table 1.

Post-operatively, in order to direct the gas bubble to the break site the patient's head was positioned so that the retinal break was uppermost for at least 5 days. Intraocular pressure was measured $6 \mathrm{~h}$ after surgery and at regular intervals during follow-up. The mean length of patient follow-up was 11 months (range 5-24 months).

\section{Results}

\section{Patient characteristics}

Thirty-one patients ( 20 men, 11 women) with a mean age of 63.4 years (range 29-81 years) underwent pneumatic retinopexy for the treatment of primary rhegmatogenous retinal detachment.

Table 2. Pre-operative features (31 eyes)

\begin{tabular}{lc}
\hline Feature & No. of eyes \\
\hline Myopia >-3 D & 8 \\
Pseudophakia & \\
$\quad$ Intact posterior capsule & 3 \\
$\quad$ Anterior chamber implant & 1 \\
Number of retinal breaks & \\
One & 27 \\
Two & 4 \\
Location of retinal breaks & ${ }^{a}$ \\
12 o'clock & \\
Superonasal & 2 \\
Superotemporal & 6 \\
Extent of detachment & 23 \\
$\quad<1$ quadrant & \\
1 to <2 quadrants & 9 \\
2 to <3 quadrants & 14 \\
3 to 4 quadrants & 5 \\
Macular detachment & 3 \\
Duration of detachment & 20 \\
$\quad<1$ week & \\
1 week to 1 month & 23 \\
$>1$ month & 3 \\
\hline
\end{tabular}

${ }^{a}$ All above horizontal.
Table 3. Anatomical and visual results (31 eyes)

\begin{tabular}{lc}
\hline Results & No. of eyes \\
\hline Reattached with pneumatic retinopexy only & 19 \\
Reattached with one subsequent operation only & 7 \\
Reattached with additional vitreoretinal procedures & 1 \\
Total reattachment & 27 \\
Macula-on detachments & \\
Unchanged post-operative acuity & 10 \\
Failed to reattach & 1 \\
Macula-off detachments & \\
$6 / 12$ or better & 9 \\
$6 / 18-6 / 60$ & 6 \\
Less than $6 / 60$ & 5 \\
\hline
\end{tabular}

Twenty-six of the 31 eyes had a single retinal break 1 clock-hour or smaller in size. The remaining had multiple small breaks within 1 clock-hour of each other except for one eye with a single large break 2 clock-hours in size. All the breaks were located in the superior 6 clock-hours of the fundus with the majority present in the superotemporal quadrant.

The detachment involved no more than two retinal quadrants in 23 eyes and the macula was detached in 20 eyes. Of the 4 pseudophakic eyes, 3 had intact posterior capsules and 1 had an anterior chamber intraocular lens implant. Other pre-operative features are summarised in Table 2.

\section{Surgical results}

Pneumatic retinopexy resulted in initial retinal reattachment in 22 eyes. In 2 patients the detachment recurred within the first month and in a third at 4 months post-operatively, giving an anatomical reattachment with one procedure in 19 out of 31 eyes (61\%) (Table 3).

The initial failures were due to the break remaining open in 4 cases, to new or missed inferior breaks in 6 cases and to the development of PVR in two cases (Table 4).

Of the 12 failures, 7 were reattached with one additional operation ( 6 had scleral buckling and 1 a vitrectomy with $\mathrm{SF}_{6}$ gas tamponade) and 1 case reattached after four vitreoretinal procedures, giving an overall reattachment rate of $87 \%$. Peripheral epiretinal membrane proliferation caused persistent but stable localised inferior tractional retinal detachments after pneumatic retinopexy in 2 eyes and PVR led to total detachment in 2 others despite multiple operations.

Of the 11 eyes presenting with macula-on retinal detachments, 10 had unchanged post-operative visual acuity and 1 failed to reattach. The post-operative visual acuity of the 20 eyes with pre-operative macular

Table 4. Complications (31 eyes)

\begin{tabular}{lc}
\hline Complication & No. of eyes \\
\hline Subconjunctival gas & 3 \\
Gas behind the lens & 3 \\
New or missed retinal breaks & 7 \\
Proliferative vitreoretinopathy & 4 \\
Late cataract & 5 \\
Macular pucker & 3 \\
\hline
\end{tabular}


detachment was $6 / 12$ or better in 9 cases, worse than $6 / 60$ in 5 cases ( 2 failed to reattach) and between $6 / 60$ and $6 / 18$ in 6 cases (Table 3 ).

\section{Complications}

Per-operative gas entrapment at the injection site between the lens and the anterior hyaloid face occurred in 3 cases (Table 4). In these cases the break remained open and the subretinal fluid failed to absorb despite posturing in the face-down position for the first $24 \mathrm{~h}$. Multiple gas bubbles ('fish eggs') were noted in 4 eyes but these coalesced forming a single bubble at first follow-up examination and did not seem to influence the outcome of the procedure. No significant increase in intraocular pressure was noted $6 \mathrm{~h}$ after the operation.

Post-operative complications included new or missed breaks in 7 eyes: all these were detected in the inferior retina within 3 weeks following the pneumatic retinopexy. One patient with no subretinal fluid had laser treatment only, 5 required a scleral buckling operation and 1 patient underwent additional vitrectomy to reattach the retina.

Increased lens opacities were noted in 5 eyes and macular pucker in 3 (Table 4).

\section{Discussion}

Our results show a similar reattachment rate following a single procedure compared with some previously published data. ${ }^{3-7}$ In a collaborative study of 100 cases, Hilton demonstrated a reattachment rate of $84 \%$ with one pneumatic retinopexy procedure only, ${ }^{9}$ while a metaanalysis review of a 100 papers reporting results on more than 1200 eyes gives an average single-operation success rate of $80 \%{ }^{8}$ Algvere et al. and most recently Han et al. reported a single operation success rate of $62 \% .{ }^{5,3}$ and a final reattachment rate of $95 \%$ and $98 \%$, respectively. Our reattachment rate of $61 \%$ with a single procedure and a final success of $87 \%$ after additional surgery are comparable to the above results. Moreover if the two cases of localised tractional detachment are considered, then the posterior reattachment rate in our series is $94 \%$.

Pseudophakia did not appear to be a poor prognostic factor in this series, as in 3 of the 4 patients with previous cataract extraction the retina reattached after pneumatic retinopexy alone. The pneumatic retinopexy failed in the patient with an anterior chamber intraocular lens implant.

Although the median age of the failed pneumatic retinopexy group is younger than that of the group with successful reattachment, these data provide no evidence of a difference in age between the two groups as assessed by the rank sum test $(p=0.143)$. This test also

demonstrated no significant difference in the duration of the detachment between the success and failure groups $(p=0.601)$. Although the extent of retinal detachment had a higher median value in patients with failed pneumatic retinopexy, the rank sum test demonstrated no significant difference in the number of quadrants affected between the success and failure groups ( $p=$ $0.165)$. Moreover we did not detect any association between macular detachment and surgical outcome as assessed by Fisher's exact test $(p=0.282)$. Contrary to previous reports ${ }^{16}$ we did not detect a gender effect as the male to female ratio was the same in both reattached and non-reattached groups after a single pneumatic retinopexy procedure. We acknowledge that failure to detect a significant difference in the extent or duration of detachments and age or sex of the patients in relation to the success of the procedure may be in part related to the small number of patients in the study.

\section{Visual acuity}

All our patients with macula-on detachments had the same or better visual acuity after pneumatic retinopexy except for the patient whose retina failed to reattach despite multiple operations. Of the 20 macula-off detachments, $45 \%$ improved to $6 / 12$ or better and $30 \%$ to between $6 / 18$ and $6 / 60$. The reason for these worse than expected post-operative visual acuities may be that 5 patients had macula-off detachments for more than 2 weeks prior to surgery.

\section{Failure of absorption of subretinal fluid}

In 4 of the 12 failed cases (13\%) the initial break remained open and subretinal fluid failed to absorb with pneumatic retinopexy alone. This incidence lies within the $1-14 \%$ range reported by other studies. $4,9,13,15,17$ The cause of the initial retinal break failing to close could be directly related to poor compliance in posturing in 1 patient only. This patient subsequently underwent multiple vitreoretinal procedures and her retina remained detached due to extensive PVR. On the other hand, initial gas entrapment between the lens and the anterior vitreous face could be responsible for nonclosure of the break in the remaining 3 patients. Inadequate gas volume or possibly excessive vitreoretinal traction on the break might also have contributed to failure.

\section{New breaks}

The high incidence $(22 \%)$ of secondary inferior retinal breaks following pneumatic retinopexy in our patients is partly responsible for the lower initial success rate of reattachment than in other series of pneumatic retinopexy. 8 Although similar figures are reported elsewhere ${ }^{4}$ it appears that the average incidence of these breaks described in larger series is $13 \%,{ }^{8}$ with most being noted within the first month after the injection of gas. New or missed retinal breaks were responsible for $6(50 \%)$ failures in our series. These breaks were all inferior, leading to retinal detachment within 1 month following pneumatic retinopexy. Of those, 5 patients were successfully managed with one additional operation and in 1 case the retina remained detached due to PVR despite multiple operations. All our patients had 
their inferior retina carefully examined with scleral indentation pre-operatively and patients with inferior vitreoretinal pathology were not offered pneumatic retinopexy. The development of new breaks is thought to be related to factors such as posterior vitreous detachment complicated by upward movement of the gas bubble, ocular motion and positioning of the patient.

\section{PVR}

An increase in vitreous flare and pigment cells has been observed as a result of subretinal fluid being squeezed through the open break by the gas bubble in pneumatic retinopexy ${ }^{18}$ and this may increase the risk of PVR developing. Our incidence of PVR (13\%) is similar to that reported by Han et al. ${ }^{3}$ and Chen et al. ${ }^{4}$ and slightly higher than in some other series..$^{8,9,13,19}$ Peripheral epiretinal membranes developed after pneumatic retinopexy in 2 patients leading to inferior tractional detachment at 1 and 4 months. The first patient underwent an additional scleral buckle operation and both had a persistent but stable and localised tractional retinal detachment. PVR led to the ultimate failure of reattachment in 2 other patients.

Our results suggest that pneumatic retinopexy can be a useful alternative to conventional rhegmatogenous retinal detachment surgery in carefully selected cases when reduced tissue trauma and quicker rehabilitation are required. The higher incidence of new inferior breaks, PVR and subsequent failures in our series and the lower single-operation success rate than with scleral buckling diminish its appeal as an apparently quick and simple procedure without the need for expensive hospitalisation. Although a failed pneumatic retinopexy is claimed not to compromise the success of further procedures nor the final visual acuity, ${ }^{3,13,14,20}$ and despite being advocated for patients unfit for more invasive surgery, this technique might be particularly unsuitable for some of these cases as it requires the patient's mental and physical co-operation and perseverance with rigorous post-operative positioning and very close postoperative follow-up. It has been our clinical impression that patients whose general health precluded a buckling procedure under general or local anaesthetic also find post-operative positioning difficult to cope with. A larger study addressing the influence of such non-ocular factors is warranted.

\section{References}

1. Hilton GF, Grizzard WS. Pneumatic retinopexy: a two-step outpatient operation without conjunctival incision. Ophthalmology 1986;93:626-41.
2. Liggett PE. Clinical experience: Doheny Eye Institute, Los Angeles, California. In: Tornambe PE, Grizzard WS, editors. Pneumatic retinopexy: a clinical symposium, 1988. Chicago: Greenwood, 1989:155-6.

3. Han DP, Mohsin NC, Guse GE, Hartz A, Tarkanian CN, et al. Comparison of pneumatic retinopexy and scleral buckling in the management of primary rhegmatogenous retinal detachment. Am J Ophthalmol 1998;126:658-68.

4. Chen JC, Robertson JE, Coonan P, et al. Results and complications of pneumatic retinopexy. Ophthalmology 1988;95:601-8.

5. Algvere P, Hallnas K, Palmqvist BM. Success and complications of pneumatic retinopexy. Am J Ophthalmol 1988;106:400-4.

6. Berrod JP, Bazard MC, Bodard E, et al. La rétinopexie pneumatique par $\mathrm{SF}_{6}$ ou $\mathrm{C}_{3} \mathrm{~F}_{8}$ : résultats et complications à propos de 56 patients. Bull Soc Ophtalmol Fr 1991;1:117-21.

7. Bochow TW, Olk RJ, Hershey JM. Pneumatic retinopexy perfluoroethane $\left(\mathrm{C}_{2} \mathrm{~F}_{6}\right)$ in the treatment of rhegmatogenous retinal detachment. Arch Ophthalmol 1992;110:1723-4.

8. Hilton GF, Tornambe PE. The Retinal Detachment Study Group. Pneumatic retinopexy: an analysis of intraoperative and postoperative complications. Retina 1991;11:285-94.

9. Hilton GF, Kelly NE, Salzano TC, et al. Pneumatic retinopexy: a collaborative report of the first 100 cases. Ophthalmology 1987;94:307-14.

10. Tornambe PE, Hilton GF, Kelly NF, Salzano TC, Wells JW, Wendel RT. Expanded indications for pneumatic retinopexy. Ophthalmology 1988;95:597-600.

11. Kreissig I, Stanowsky A, Lincoff $\mathrm{H}$, Richard G. The treatment of difficult retinal detachments with an expanding gas bubble without vitrectomy. Graefes Arch Clin Exp Ophthalmol 1986;224:51-4.

12. McAllister IL, Zeggara H, Meyers SM, Gutman F. Treatment of retinal detachments with multiple breaks by pneumatic retinopexy. Arch Ophthalmol 1987;105:913-6.

13. Tornambe PE, Hilton GF. The Retinal Detachment Study Group. Pneumatic retinopexy: a multicenter randomised controlled clinical trial comparing pneumatic retinopexy with scleral buckling. Ophthalmology 1989;96:772-84.

14. Tornambe PE, Hilton GF. The Retinal Detachment Study Group. Pneumatic retinopexy: a two year follow-up study of the multicenter trial comparing pneumatic retinopexy with scleral buckling. Ophthalmology 1991;98:1115-23.

15. McAllister IL, Meyers SF, Zegarra H, Gutman F, Zakov ZN, Beck GJ. Comparison of pneumatic retinopexy with alternative surgical techniques. Ophthalmology 1988;95:877-83.

16. Grizzard WS, Hilton GF, Hammer ME, Taren D, Brinton DA. Pneumatic retinopexy failures: cause, prevention, timing and management. Ophthalmology 1995,102:929-36.

17. Chan CK, Wessels IF. Delayed subretinal fluid absorption after pneumatic retinopexy. Ophthalmology 1989;96:1691-700.

18. Griffith PG, Richardson J. Causes of proliferative retinopathy following pneumatic retinopexy. Arch Ophthalmol 1990;108:1515.

19. Trillo M, Facino M, Terrile R, et al. Treatment of uncomplicated cases of rhegmatogenous retinal detachment with an expanding gas bubble. Ophthalmologica 1993;207:140-3.

20. Ambler JS, Meyers SM, Zegarra H, Paranandi L. Reoperations and visual results after failed pneumatic retinopexy. Ophthalmology 1990;97:786-90. 